\title{
Human Capital Accumulation in R\&D-based Growth Models
}

C. Mattalia

Discussion Paper 2005-46

\section{Département des Sciences Économiques de l'Université catholique de Louvain}




\title{
Human Capital Accumulation in R\&D-based Growth Models
}

\author{
Claudio Mattalia*
}

September 2005

\begin{abstract}
This paper considers a multi-sectoral endogenous growth model, that reproduces the essential aspects of an "ICT-based economy", in which a central role is played by human capital accumulation. Indeed, households also invest in human capital through schooling, and this turns out to be the true engine of growth. Furthermore, this model displays no scale effect and the simulations allow to get interesting results concerning the link between market power and growth, the presence of "imbalance effects" and the consequences of different types of subsidies.
\end{abstract}

Keywords: Information Technology, Endogenous Growth, Imbalance Effect.

Journal of Economic Literature: E22, O40, C63.

*Dipartimento di Statistica e Matematica Applicata alle Scienze Umane "Diego de Castro", Università degli Studi di Torino (Italy) and IRES, Université catholique de Louvain, Louvain-la-Neuve (Belgium). E-mail: claudio.mattalia@unito.it. The author thanks Raouf Boucekkine for very useful remarks and suggestions. We are grateful for the financial support from the Belgian French Community's program "Action de recherches concertée" 03/08 -302. 


\section{Introduction}

This paper considers a multi-sectoral endogenous growth model, that is able to reproduce the most important aspects of an "ICT-based economy", in which a central role is played by human capital accumulation. Indeed, one of the aspects of the so-called "new economy" is that human capital can be of great importance, since education is crucial in acquiring the knowledge necessary to use the new technologies, and at the same time an increase in ICT makes it easier to accumulate human capital. For these reasons it is important to derive a model that captures these aspects, and the one considered here in particular extends the model proposed in a previous paper (Mattalia, 2002), and introduces the choice of the households to invest in human capital, by devoting a fraction of time to schooling activity. This human capital accumulation then turns out to be the true engine of growth of the model, that therefore has important differences with respect to the one presented in the previous work. Furthermore, in this case the analysis shows that the growth rate of output doesn't depend neither on the absolute dimension of the economy nor on the population growth, hence the model has the important property according to which it doesn't display any scale effect.

Numerical simulations can then be implemented in order to study the short run behavior of the economy in different situations, and in this way it is possible to obtain important results with reference to two aspects. On the one hand, a positive relationship between market power and growth emerges: when the economy is characterized by an increase in the monopoly power (that is a reduction in the competitivity of the system), a higher growth rate of output arises. On the other hand, "imbalance effects" arise with reference to some variables (in particular the ratio between physical and human capital, the ratio between physical capital and $R \& D$ expenditure and the ratio between human capital and $R \& D$ expenditure): when the initial value of these variables is different from the steady state value (in particular, lower) the growth rate of the economy is higher than the rate we have in correspondence of the steady state value of the variables considered. This is of interest, for instance, with reference to the behavior of an underdeveloped economy, that initially has an amount of human capital or of investment in R\&D lower than the optimal one.

Finally, some extensions of the model are considered, introducing the notion of "broad output" (following Barro and Sala-i-Martin, 1995) and con- 
sidering different types of subsidies that can be introduced (on $\mathrm{R} \& \mathrm{D}$, on physical capital accumulation, on human capital accumulation). The results are that, also when the more general notion of broad output is considered, imbalance effects are present, and that, contrary to the other types of subsidies, the introduction of subsidies on human capital accumulation has (in the short run) a positive effect on growth. A further extension considers the productivity of human capital as a function of technological progress, in order to establish a link between human capital accumulation and technological progress (that is typical of ICT, since new technologies can allow people to be more educated), and also in this case the presence of imbalance effects is confirmed.

The model presented here builds on Romer (1990) and Boucekkine and de la Croix (2003) for the general structure, but departs from them in some respects. In particular, the $\mathrm{R} \& \mathrm{D}$ sector assumes the "lab-equipment" specification (Rivera-Batiz and Romer, 1991), and a human capital accumulation activity of the kind introduced by Lucas (1988) is considered. The reason is that, together with the embodied nature of technological progress, the important role of the $\mathrm{R} \& \mathrm{D}$ sector and the link between innovation and market power (all elements that are present in the model introduced in the previous work), an ICT economy can be characterized also by an important role of human capital accumulation (for the reasons cited above).

The paper is organized as follows. Section 2 describes the model with the different sectors that characterize the economy. Section 3 derives the equilibrium conditions, the balanced growth path and the steady state system, with the corresponding analytical results. Section 4 shows the results of numerical simulations on a calibrated version of the model and derives the main findings. Section 5 considers possible extensions of the model and Section 6 concludes.

\section{The model}

The model has the same general structure of the one presented in the previous paper, in particular it is a multi-sectoral model written in discrete time with infinite horizon, endogenous growth and horizontal differentiation. Furthermore, technological progress is mainly embodied (new softwares need new hardware to work efficiently) and the innovators have a market power 
represented by copyrights. The main differences with respect to the model of the previous work are represented by the fact that human capital is employed as a production factor in the final good sector and in the intermediate good sector, and by the fact that the representative household also invests in human capital, by devoting a fraction of time to non-productive activities (schooling). We now illustrate briefly the different sectors that characterize the economy, with the corrisponding optimality conditions that arise from the optimization problems solved by the agents in these sectors.

\subsection{The final good sector}

This sector produces the final good using efficient capital (bought from the equipment sector) and human capital, according through a Cobb-Douglas technology:

$$
Y_{t}=z_{t} K_{t}^{\alpha} H_{Y, t}^{1-\alpha} \quad \alpha \in[0,1]
$$

where $z_{t}$ represents total factor productivity, $K_{t}$ is the physical capital and $H_{Y, t}$ is the human capital used in the final good sector. The stock of physical capital is then defined as:

$$
K_{t}=\sum_{s=-\infty}^{t} E_{s}(1-\delta)^{t-s}
$$

where $E_{s}$ is the efficient capital bought from the equipment sector at time $s$ and $\delta$ is the physical depreciation rate (constant).

The discounted profits of investing $E_{t}$ in efficient capital are given by:

$$
\pi_{t}=\sum_{s=t}^{\infty}\left[Y_{s}-w_{s} H_{Y, s}\right] R_{t}^{s}-d_{t} E_{t}
$$

where $R_{t}^{s}$ is the discount factor at time $s, w_{s}$ is the wage at time $s$ and $d_{t}$ is the price of efficient capital at time $t$. The representative firm chooses efficient capital and human capital in order to maximize its discounted profits taking prices as given and subject to its technological constraints:

$$
\begin{aligned}
& \max _{E_{t},\left\{H_{Y, s}\right\}_{s=t}^{\infty}} \pi_{t} \\
& \text { s.t. } \quad(1),(2)
\end{aligned}
$$


and the first-order conditions lead to:

$$
H_{Y, t}=\left(\frac{(1-\alpha) z_{t}}{w_{t}}\right)^{\frac{1}{\alpha}} K_{t}
$$

that is the demand for human capital by the final good sector.

\subsection{The equipment sector}

This sector produces efficient capital (sold to the final good sector) using physical capital (hardware) bought from the final good producers and immaterial capital (software) bought from the intermediate good producers. Efficient capital is produced with a constant return to scale technology:

$$
E_{t}=e_{t} Q_{t}^{\lambda} I_{t}^{1-\lambda} \quad \lambda \in(0,1)
$$

where $e_{t}$ is a productivity variable (that represents embodied technological progress), $I_{t}$ is physical capital (hardware) and $Q_{t}$ is immaterial capital (software), that is built from a series of specialized intermediate goods (horizontal differentiation):

$$
Q_{t}=\left(\int_{0}^{n_{t}} x_{i, t}^{\frac{\sigma-1}{\sigma}} d i\right)^{\frac{\sigma}{\sigma-1}}
$$

where $n_{t}$ is the number of varieties of intermediate input available in $t, x_{i, t}$ is the quantity of intermediate input of variety $i$ used in $t$ and $\sigma>1$ is the elasticity of substitution between two varieties.

The profits of the equipment sector at time $t$ are:

$$
\pi_{t}^{\prime}=d_{t} E_{t}-I_{t}-\int_{0}^{n_{t}} p_{i, t} x_{i, t} d i
$$

where $p_{i, t}$ is the price of software of variety $i$ at time $t$. The representative firm chooses the investment in physical capital and in immaterial capital in order to maximize profits taking prices as given and subject to its technological constraints:

$$
\begin{aligned}
& \max _{I_{t}, x_{i, t}} \pi_{t}^{\prime} \\
& \text { s.t. }
\end{aligned}
$$


and the first-order conditions lead to:

$$
x_{i, t}=\left(\frac{\phi}{q_{t}}\right)^{\sigma} Q_{t} p_{i, t}^{-\sigma}
$$

(where $\phi=\frac{\lambda}{1-\lambda}$ and $q_{t}=\frac{Q_{t}}{I_{t}}$ ) that is the demand for intermediate input $i$ by the equipment sector at time $t$.

\subsection{The intermediate good sector}

This sector produces immaterial capital (sold to the equipment sector) and it researches for new varieties, in order to expand the range of software (horizontal differentiation).

The variety $i$ of software is produced according to a linear technology that uses human capital as the only input:

$$
x_{i, t}=\tau_{t} H_{i, t}
$$

where $H_{i, t}$ is the human capital used in the intermediate good sector and $\tau_{t}$ represents productivity of human capital. The producer behaves monopolistically (since market power is given by the presence of copyrights which have an infinite lifetime) and its profit is:

$$
\pi_{i, t}^{\prime \prime}=p_{i, t} x_{i, t}-w_{t} H_{i, t}=\left(p_{i, t}-\frac{w_{t}}{\tau_{t}}\right) x_{i, t}
$$

The price of output is chosen so as to maximize this profit subject to the demand formulated by the equipment sector, hence the problem solved by the firm is:

$$
\begin{array}{ll}
\max _{p_{i, t}} & \pi_{i, t}^{\prime \prime} \\
\text { s.t. } & \quad(6)
\end{array}
$$

and the first-order condition leads to:

$$
p_{i, t}=\left(\frac{\sigma}{\sigma-1}\right) \frac{w_{t}}{\tau_{t}} \quad \forall i \in\left[0, n_{t}\right]
$$

i.e. the output price is a mark-up over unit human capital cost. 


\subsection{The $\mathrm{R} \& \mathrm{D}$ sector}

The intermediate good sector also researches for new varieties of immaterial capital, in order to expand their range. Assuming the "lab-equipment" specification of $\mathrm{R} \& \mathrm{D}$, according to which the cost to create a new variety of software is equal to $\eta$ units of $Y$, there will be entry of new firms in the economy until this cost is equal to the discounted flow of profits linked to one invention:

$$
\eta=\sum_{z=t}^{\infty} R_{t}^{z} \pi_{i, z}^{\prime \prime}=\sum_{z=t}^{\infty} R_{t}^{z} \frac{1}{\sigma-1} \frac{w_{z}}{\tau_{z}} x_{i, z}
$$

that is the free-entry condition.

\subsection{Household behavior}

In this version of the model, the representative household consumes, saves for future consumption, supplies human capital for productive activities and accumulates human capital by devoting a fraction of its time to non-productive activities (schooling). The utility of the representative household at time 0 is:

$$
u_{0}=\sum_{t=0}^{\infty} \rho^{t} \ln C_{t}
$$

i.e. it is the discounted sum of instantaneous utilities from 0 to $\infty$, where $\rho$ is the psychological discount factor and the instantaneous utility function is logarithmic. The budget constraint faced by the household is:

$$
A_{t}=\left(1+r_{t}\right) A_{t-1}+w_{t} u_{t} H_{t-1}-C_{t}
$$

where $A_{t}$ represents the assets held by the household at time $t, H_{t}$ is the total human capital and $u_{t}$ is the fraction of time devoted to productive activities. The accumulation of human capital is then described by the following equation:

$$
H_{t}=\left[1+\varepsilon_{t}\left(1-u_{t}\right)\right] H_{t-1}
$$

where $\varepsilon_{t}$ is the productivity of schooling and $1-u_{t}$ is the fraction of time devoted to non-productive activities (schooling).

The problem solved by the representative household is then:

$$
\begin{aligned}
& \max _{\left\{C_{t}, u_{t}, A_{t}, H_{t}\right\}_{t=0}^{\infty}} u_{0} \\
& \text { s.t. } \quad(9),(10)
\end{aligned}
$$


and the first-order conditions lead to:

$$
\begin{gathered}
\frac{C_{t+1}}{C_{t}}=\left(1+r_{t+1}\right) \rho \\
\frac{w_{t+1}}{w_{t}}=\frac{1+r_{t+1}}{1+\varepsilon_{t+1}} \cdot \frac{\varepsilon_{t+1}}{\varepsilon_{t}}
\end{gathered}
$$

together with the corresponding transversality conditions.

\section{The equilibrium}

The equilibrium of the economy in the model considered is determined by the equilibrium on the human capital market and on the final good market.

Equilibrium on the human capital market implies that human capital used in productive activities is employed either in the final good sector or in the intermediate good sector, i.e.:

$$
H_{Y, t}+\int_{0}^{n_{t}} H_{i, t} d i=u_{t} H_{t-1}
$$

while equilibrium on the final good market implies that output is employed for consumption, investment and research, i.e.:

$$
Y_{t}=C_{t}+I_{t}+\eta \triangle n_{t}
$$

where $\eta \triangle n_{t}$ is the cost of research for new varieties.

\subsection{The equilibrium conditions}

The equilibrium conditions summarize the first order optimality conditions and the market equilibrium relationships derived above.

In particular, the demand for human capital by the final good sector is given by equation (3), while the demand for human capital by the intermediate good sector can be obtained from equations (6), (7) and (8) and is given by:

$$
\int_{0}^{n_{t}} H_{i, t} d i=n_{t}\left(\frac{\sigma-1}{\sigma}\right)^{\sigma}\left(\frac{\phi}{w_{t}}\right)^{\sigma} q_{t}^{1-\sigma} I_{t} \tau_{t}^{\sigma-1}
$$


and therefore the equilibrium on the human capital market, given by equation (13), is:

$$
\left(\frac{(1-\alpha) z_{t}}{w_{t}}\right)^{\frac{1}{\alpha}} K_{t}+n_{t}\left(\frac{\sigma-1}{\sigma}\right)^{\sigma}\left(\frac{\phi}{w_{t}}\right)^{\sigma} q_{t}^{1-\sigma} I_{t} \tau_{t}^{\sigma-1}=u_{t} H_{t-1}
$$

The equilibrium on the final good market can be obtained from equations (1), (3) and (14) and is:

$$
z_{t}^{\frac{1}{\alpha}} K_{t}\left(\frac{1-\alpha}{w_{t}}\right)^{\frac{1-\alpha}{\alpha}}=C_{t}+I_{t}+\eta \triangle n_{t}
$$

The law of motion for $q_{t}$ can be obtained substituting the expression (3) in the first-order condition of the problem of the representative firm in the final good sector, using the definition of $K_{s}$ and replacing the value of $d_{t}$ from the first-order condition of the problem of the representative firm in the equipment sector, and is:

$$
\alpha z_{t}^{\frac{1}{\alpha}}(1-\lambda) e_{t} q_{t}^{\lambda}\left(\frac{1-\alpha}{w_{t}}\right)^{\frac{1-\alpha}{\alpha}}=1-\left(\frac{1-\delta}{1+r_{t+1}}\right)\left(\frac{e_{t}}{e_{t+1}}\right)\left(\frac{q_{t}}{q_{t+1}}\right)^{\lambda}
$$

while the law of motion for $H_{t}$ is given by equation (10):

$$
H_{t}=\left[1+\varepsilon_{t}\left(1-u_{t}\right)\right] H_{t-1}
$$

The optimal consumption is then given by equation (11):

$$
\frac{C_{t+1}}{C_{t}}=\left(1+r_{t+1}\right) \rho
$$

and the optimal wage by equation (12):

$$
\frac{w_{t+1}}{w_{t}}=\frac{1+r_{t+1}}{1+\varepsilon_{t+1}} \cdot \frac{\varepsilon_{t+1}}{\varepsilon_{t}}
$$

The accumulation rule of capital is obtained from equations (2) and (4) and is:

$$
K_{t}=(1-\delta) K_{t-1}+e_{t} q_{t}^{\lambda} I_{t}
$$

while from equations (5), (6) and (8) it is possible to get:

$$
\frac{w_{t} q_{t}}{\tau_{t} \phi}=n_{t}^{\frac{1}{\sigma-1}}\left(\frac{\sigma-1}{\sigma}\right)
$$


that links the embodied technological progress to the expansion in the varieties of intermediate products.

Finally, the free-entry condition can be rewritten as:

$$
\eta=\frac{\phi^{\sigma}}{\tau_{t}^{1-\sigma}(\sigma-1)^{1-\sigma} \sigma^{\sigma}} \sum_{z=t}^{\infty} R_{t}^{z} w_{z}^{1-\sigma} q_{z}^{1-\sigma} I_{z}
$$

and then:

$$
\eta R_{t}^{t+1}=\frac{\phi^{\sigma}}{\tau_{t}^{1-\sigma}(\sigma-1)^{1-\sigma} \sigma^{\sigma}} \sum_{z=t+1}^{\infty} R_{t}^{z} w_{z}^{1-\sigma} q_{z}^{1-\sigma} I_{z}
$$

The two expressions for $t$ and $t+1$ are therefore:

$$
\begin{gathered}
\frac{\tau_{t}^{1-\sigma}(\sigma-1)^{1-\sigma} \sigma^{\sigma}}{\phi^{\sigma}} \eta=\sum_{z=t}^{\infty} R_{t}^{z} w_{z}^{1-\sigma} q_{z}^{1-\sigma} I_{z} \\
\frac{\tau_{t}^{1-\sigma}(\sigma-1)^{1-\sigma} \sigma^{\sigma}}{\phi^{\sigma}} \eta R_{t}^{t+1}=\sum_{z=t+1}^{\infty} R_{t}^{z} w_{z}^{1-\sigma} q_{z}^{1-\sigma} I_{z}
\end{gathered}
$$

and subtracting the second from the first:

$$
\frac{\tau_{t}^{1-\sigma}(\sigma-1)^{1-\sigma} \sigma^{\sigma}}{\phi^{\sigma}} \cdot \frac{\eta r_{t+1}}{1+r_{t+1}}=w_{t}^{1-\sigma} q_{t}^{1-\sigma} I_{t}
$$

These results are summarized in the following Proposition:

Proposition 1 Given the initial conditions $K_{-1}, H_{-1}$ and $n_{-1}$ an equilibrium is a path:

$$
\left\{w_{t}, q_{t}, C_{t}, I_{t}, K_{t}, H_{t}, n_{t}, u_{t}, r_{t+1}\right\}_{t \geq 0}
$$

that satisfies the equations (15) - (23) shown above.

\subsection{The balanced growth path}

After the characterization of the equilibrium, the balanced growth path of the model can be analyzed. In this case we assume that the variables:

$$
z_{t}, e_{t}, \tau_{t}, \varepsilon_{t}, r_{t}, u_{t}
$$

are constant in the long term, while each endogenous variable grows at a constant rate along a balanced growth path (hence, if $\bar{x}$ is the initial level 
of the variable $x$ and $g_{x}$ is the growth factor of the same variable, we have $\left.x_{t}=\bar{x} g_{x}^{t}\right)$. In particular, the growth factors we are interested in are:

$$
g_{w}, g_{q}, g_{C}, g_{I}, g_{K}, g_{H}, g_{n}
$$

and since a balanced growth path must satisfy the equations (15) - (23), we have the following nine restrictions among these growth factors (to obtain them it is sufficient to rewrite the equilibrium equations substituting the generic variable $x_{t}$ with the expression $\left.\bar{x} g_{x}^{t}\right)$ :

$$
\begin{gathered}
g_{K}\left(g_{w}\right)^{-\frac{1}{\alpha}}=\frac{g_{n}}{\left(g_{w}\right)^{\sigma}}\left(g_{q}\right)^{1-\sigma} g_{I}=g_{H} \\
g_{Y}=g_{C}=g_{I}=g_{n}=g_{K}\left(g_{w}\right)^{-\frac{1-\alpha}{\alpha}} \\
\left(g_{q}\right)^{\lambda}\left(g_{w}\right)^{-\frac{1-\alpha}{\alpha}}=1 \\
g_{H}=[1+\varepsilon(1-u)] \\
g_{C}=(1+r) \rho \\
g_{w}=\frac{1+r}{1+\varepsilon} \\
g_{K}=\left(g_{q}\right)^{\lambda} g_{I} \\
g_{w} g_{q}=g_{n}^{\frac{1}{\sigma-1}} \\
\left(g_{w}\right)^{1-\sigma}\left(g_{q}\right)^{1-\sigma} g_{I}=1
\end{gathered}
$$

In correspondence of a balanced growth path, the various growth factors must therefore satisfy the restrictions expressed by equations (24) - (32), and using these restrictions in this case it is possible to determine explicitely the different growth factors. In fact, first of all from (26) we have:

$$
g_{w}=\left(g_{q}\right)^{\frac{\lambda \alpha}{1-\alpha}}
$$

From (31) we get:

$$
g_{n}=\left(g_{q}\right)^{\frac{(\sigma-1)(1-\alpha+\lambda \alpha)}{1-\alpha}}=g_{Y}=g_{C}=g_{I}
$$

From (30) we obtain:

$$
g_{K}=\left(g_{q}\right)^{\lambda+\frac{(\sigma-1)(1-\alpha+\lambda \alpha)}{1-\alpha}}
$$


and the same result can be obtained from (25).

From (24) we have:

$$
g_{H}=\left(g_{q}\right)^{\frac{\sigma(1-\alpha+\lambda \alpha)-1+\alpha-2 \lambda \alpha}{1-\alpha}}
$$

while (32) is satisfied.

From (28) we then get:

$$
r=\frac{g_{C}}{\rho}-1=\frac{\left(g_{q}\right)^{\frac{(\sigma-1)(1-\alpha+\lambda \alpha)}{1-\alpha}}}{\rho}-1
$$

and finally from (29) we have:

$$
\left(g_{q}\right)^{\frac{\lambda \alpha}{1-\alpha}}=\frac{\left(g_{q}\right)^{\frac{(\sigma-1)(1-\alpha+\lambda \alpha)}{1-\alpha}}}{\rho(1+\varepsilon)}
$$

that enables to obtain explicitely the value of $g_{q}$ :

$$
g_{q}=[\rho(1+\varepsilon)]^{\frac{1-\alpha}{\sigma(1-\alpha+\lambda \alpha)-1+\alpha-2 \lambda \alpha}}
$$

and then the values of the other growth factors as a function of the different parameters of the model $(\alpha, \lambda, \sigma, \rho, \varepsilon)$, since the seven unknowns of the problem $\left(g_{w}, g_{q}, g_{Y}, g_{K}, g_{H}, r, u\right)$ are related by a system of seven equations (the equations (24), (26), (27), (28), (29), (30), (31) while (25) and (32) are redundant). Furthermore, from (27) we get (using the value of $g_{H}$ determined from the value just found for $g_{q}$, and that turns out to be $g_{H}=\rho(1+\varepsilon)$ ):

$$
u=1-\frac{g_{H}-1}{\varepsilon}=\frac{(1+\varepsilon)(1-\rho)}{\varepsilon}
$$

and since $0<u<1$ this requires $\frac{1}{1+\varepsilon}<\rho<1$.

The results obtained are expressed in the following Proposition:

Proposition 2 In the model considered with human capital accumulation, the growth factors of the different variables are the following:

$$
\begin{array}{r}
g_{Y}=g_{C}=g_{I}=g_{n}=[\rho(1+\varepsilon)]^{\frac{1}{\omega_{4}}} \\
g_{q}=[\rho(1+\varepsilon)]^{\frac{\omega_{1}}{\omega_{2} \omega_{4}}}
\end{array}
$$




$$
\begin{aligned}
& g_{w}=[\rho(1+\varepsilon)]^{\frac{1}{\omega_{2} \omega_{4}}} \\
& g_{K}=[\rho(1+\varepsilon)]^{\frac{\omega_{3}}{\omega_{4}}} \\
& g_{H}=\rho(1+\varepsilon)
\end{aligned}
$$

where:

$$
\begin{array}{cc}
\omega_{1}=\frac{1-\alpha}{\lambda \alpha} & \omega_{2}=\frac{(\sigma-1)(1-\alpha+\lambda \alpha)}{\lambda \alpha} \\
\omega_{3}=\frac{\lambda(1-\alpha)}{(\sigma-1)(1-\alpha+\lambda \alpha)}+1 & \omega_{4}=\frac{\sigma(1-\alpha+\lambda \alpha)-1+\alpha-2 \lambda \alpha}{(\sigma-1)(1-\alpha+\lambda \alpha)}
\end{array}
$$

Furthermore, the optimal long run value of $u$ (the fraction of time devoted to productive activities) is given by:

$$
u^{*}=\frac{(1+\varepsilon)(1-\rho)}{\varepsilon}
$$

and since $0<u^{*}<1$ this requires $\frac{1}{1+\varepsilon}<\rho<1$.

From the results obtained it turns out that, in this case, along a balanced growth path the growth factors of the different quantities depend only on the parameters $\alpha, \lambda, \sigma, \rho, \varepsilon$ (i.e. technological and preference parameters). In particular, the growth rate of production doesn't depend neither on the absolute dimension of the economy (i.e. its total human capital stock) nor on the population growth (that is equal to zero), hence we don't have any scale effect, that is an important property of this model.

\subsection{The stationarized dynamic system and the steady state system}

Even if the various growth factors can be determined explicitely, the equilibrium system in terms of levels formed by equations $(15)-(23)$ is undetermined, in fact rewriting these equations by substituting the generic variable $x_{t}$ with the expression $\bar{x} g_{x}^{t}$ we get 9 equations for 10 unknowns $(\bar{w}, \bar{q}, \bar{C}$, $\bar{I}, \bar{K}, \bar{H}, \bar{n}, \bar{r}, \bar{u}, g)$. In order to find a solution is therefore necessary to stationarize the system by means of some auxiliary variables. In particular, the following stationary variables can be used: 


$$
\begin{aligned}
& \widehat{q}_{t}=\frac{q_{t}}{w_{t}^{\omega_{1}}} \quad \widehat{C}_{t}=\frac{C_{t}}{w_{t}^{\omega_{2}}} \quad \widehat{I}_{t}=\frac{I_{t}}{w_{t}^{\omega_{2}}} \quad \widehat{K}_{t}=\frac{K_{t}}{n_{t}^{\omega_{3}}} \\
& \widehat{H}_{t}=\frac{H_{t}}{n_{t}^{\omega_{4}}} \quad \widehat{n}_{t}=\frac{n_{t}}{w_{t}^{\omega_{2}}} \quad g_{t}=\frac{n_{t}}{n_{t-1}} \quad r_{t} \quad u_{t}
\end{aligned}
$$

where $\omega_{1}, \omega_{2}, \omega_{3}, \omega_{4}$ are the expressions reported above. In this way it is possible to get the stationarized dynamic system that corresponds to the equations (15) - (23) and that is expressed by the following equations:

$$
\begin{gathered}
\left((1-\alpha) z_{t}\right)^{\frac{1}{\alpha}} \widehat{K}_{t} \widehat{n}_{t}^{\omega_{3}-\omega_{4}}+\left(\frac{\sigma-1}{\sigma}\right)^{\sigma} \phi^{\sigma} \tau_{t}^{\sigma-1} \widehat{n}_{t}^{1-\omega_{4}} \widehat{q}_{t}^{1-\sigma} \widehat{I}_{t}=u_{t} \widehat{H}_{t-1} g_{t}^{-\omega_{4}} \\
z_{t}^{\frac{1}{\alpha}} \widehat{K}_{t} \widehat{n}_{t}^{\omega_{3}}(1-\alpha)^{\frac{1-\alpha}{\alpha}}=\widehat{C}_{t}+\widehat{I}_{t}+\eta \widehat{n}_{t}\left(1-\frac{1}{g_{t}}\right) \\
\alpha z_{t}^{\frac{1}{\alpha}}(1-\lambda) e_{t} \widehat{q}_{t}^{\lambda}(1-\alpha)^{\frac{1-\alpha}{\alpha}}+\frac{1-\delta}{1+r_{t+1}}\left(\frac{e_{t}}{e_{t+1}}\right)\left(\frac{\widehat{q}_{t}}{\widehat{q}_{t+1}}\right)^{\lambda}\left(\frac{\widehat{n}_{t+1}}{\widehat{n}_{t} g_{t+1}}\right)^{\omega_{3}-1}=1 \\
\widehat{H}_{t} g_{t}^{\omega_{4}}=\left[1+\varepsilon_{t}\left(1-u_{t}\right)\right] \widehat{H}_{t-1} \\
\frac{\widehat{C}_{t+1}}{\widehat{C}_{t}} \cdot \frac{\widehat{n}_{t}}{\widehat{n}_{t+1}} g_{t+1}=\left(1+r_{t+1}\right) \rho \\
\left(\frac{\widehat{n}_{t}}{\widehat{n}_{t+1}} g_{t+1}\right)^{\frac{1}{\omega_{2}}}=\frac{1+r_{t+1}}{1+\varepsilon_{t+1}} \cdot \frac{\varepsilon_{t+1}}{\varepsilon_{t}} \\
\widehat{K}_{t}-(1-\delta) \widehat{K}_{t-1} g_{t}^{-\omega_{3}}=e_{t} \widehat{q}_{t}^{\lambda} \widehat{I}_{t} \widehat{n}_{t}^{-\omega_{3}} \\
\frac{\widehat{q}_{t}}{\tau_{t} \phi}=\widehat{n}_{t}^{\frac{1}{\sigma-1}}\left(\frac{\sigma-1}{\sigma}\right) \\
\frac{\tau_{t}^{1-\sigma}(\sigma-1)^{1-\sigma} \sigma^{\sigma}}{\phi^{\sigma}} \cdot \frac{\eta r_{t+1}}{1+r_{t+1}}=\widehat{q}_{t}^{1-\sigma} \widehat{I}_{t}
\end{gathered}
$$

In the same way it is then possible to obtain the steady state system corresponding to the stationarized one, defining the variables:

$$
\begin{aligned}
& \widehat{q}=\frac{\bar{q}}{\bar{w}^{\omega_{1}}} \quad \widehat{C}=\frac{\bar{C}}{\bar{w}^{\omega_{2}}} \quad \widehat{I}=\frac{\bar{I}}{\bar{w}^{\omega_{2}}} \quad \widehat{K}=\frac{\bar{K}}{\bar{n}^{\omega_{3}}} \\
& \widehat{H}=\frac{\bar{H}}{\bar{n}^{\omega} 4} \quad \widehat{n}=\frac{\bar{n}}{\bar{w}^{\omega} 2} \quad g=g_{n} \quad r \quad u
\end{aligned}
$$


and writing:

$$
\lim _{t \rightarrow+\infty} z_{t}=z \quad \lim _{t \rightarrow+\infty} e_{t}=e \quad \lim _{t \rightarrow+\infty} \tau_{t}=\tau \quad \lim _{t \rightarrow+\infty} \varepsilon_{t}=\varepsilon
$$
tions:

The stationarized steady state system is now given by the following equa-

$$
\begin{aligned}
& ((1-\alpha) z)^{\frac{1}{\alpha}} \widehat{K} \widehat{n}^{\omega_{3}-\omega_{4}}+\left(\frac{\sigma-1}{\sigma}\right)^{\sigma} \phi^{\sigma} \tau^{\sigma-1} \widehat{n}^{1-\omega_{4}} \widehat{q}^{1-\sigma} \widehat{I}=u \widehat{H} g^{-\omega_{4}}
\end{aligned}
$$

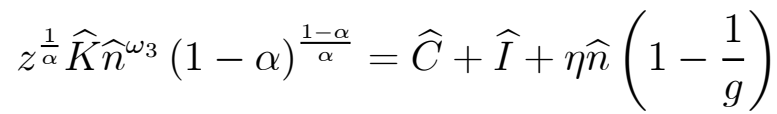

$$
\begin{aligned}
& \alpha z^{\frac{1}{\alpha}}(1-\lambda) e \widehat{q}^{\lambda}(1-\alpha)^{\frac{1-\alpha}{\alpha}}+\frac{1-\delta}{1+r} g^{1-\omega_{3}}=1 \\
& g^{\omega_{4}}=[1+\varepsilon(1-u)] \\
& g=(1+r) \rho \\
& g^{\frac{1}{\omega_{2}}}=\frac{1+r}{1+\varepsilon} \\
& \widehat{K}\left[1-(1-\delta) g^{-\omega_{3}}\right] \widehat{n}^{\omega_{3}}=e \widehat{q}^{\lambda} \widehat{I} \\
& \tau \phi\left(\frac{\sigma-1}{\sigma}\right) \widehat{n}^{\frac{1}{\sigma-1}}=\widehat{q} \\
& \frac{\tau^{1-\sigma}(\sigma-1)^{1-\sigma} \sigma^{\sigma}}{\phi^{\sigma}} \cdot \frac{\eta r}{1+r}=\widehat{q}^{1-\sigma} \widehat{I}
\end{aligned}
$$

This is a system of 9 equations with 9 unknowns $(\widehat{q}, \widehat{C}, \widehat{I}, \widehat{K}, \widehat{H}, \widehat{n}, g, r$, $u$ ) that can be solved explicitely. Indeed, first of all it is possible to obtain functions expressing the long run levels $(\widehat{q}, \widehat{C}, \widehat{I}, \widehat{K}, \widehat{H}, \widehat{n}, r, u)$ exclusively in terms of $g$, then in this case it is also possible to get an explicit expression for $g$ (depending only on the parameters of the model).

First of all, from equation (37) we have:

$$
r=\Psi_{r}(g)=\frac{g}{\rho}-1
$$

From (36) we then have:

$$
u=\Psi_{u}(g)=1-\frac{g^{\omega_{4}}-1}{\varepsilon}
$$


while from (35) we get:

$$
\widehat{q}=\Psi_{\widehat{q}}(g)=\left(\frac{1-\frac{1-\delta}{1+\Psi_{r}(g)} g^{1-\omega_{3}}}{\alpha z^{\frac{1}{\alpha}}(1-\lambda) e(1-\alpha)^{\frac{1-\alpha}{\alpha}}}\right)^{\frac{1}{\lambda}}
$$

From (40) we obtain:

$$
\widehat{n}=\Psi_{\widehat{n}}(g)=\left(\frac{\tau \phi}{\Psi_{\widehat{q}}(g)} \cdot \frac{\sigma-1}{\sigma}\right)^{1-\sigma}
$$

while from (41) we have:

$$
\widehat{I}=\Psi_{\widehat{I}}(g)=\frac{\tau^{1-\sigma}(\sigma-1)^{1-\sigma} \sigma^{\sigma}}{\phi^{\sigma}} \cdot \frac{\eta \Psi_{r}(g)}{1+\Psi_{r}(g)} \cdot \frac{1}{\left(\Psi_{\widehat{q}}(g)\right)^{1-\sigma}}
$$

and from (39) we get:

$$
\widehat{K}=\Psi_{\widehat{K}}(g)=\frac{e}{1-(1-\delta) g^{-\omega_{3}}}\left(\Psi_{\widehat{q}}(g)\right)^{\lambda} \frac{\Psi_{\widehat{I}}(g)}{\left(\Psi_{\widehat{n}}(g)\right)^{\omega_{3}}}
$$

From (34) we have:

$$
\widehat{C}=\Psi_{\widehat{C}}(g)=z^{\frac{1}{\alpha}} \Psi_{\widehat{K}}(g)\left(\Psi_{\widehat{n}}(g)\right)^{\omega_{3}}(1-\alpha)^{\frac{1-\alpha}{\alpha}}-\Psi_{\widehat{I}}(g)-\eta\left(1-\frac{1}{g}\right) \Psi_{\widehat{n}}(g)
$$

and finally from (33) we have:

$$
\begin{aligned}
\widehat{H}= & \Psi_{\widehat{H}}(g)=\frac{g^{\omega_{4}}}{u}\left[((1-\alpha) z)^{\frac{1}{\alpha}} \Psi_{\widehat{K}}(g)\left(\Psi_{\widehat{n}}(g)\right)^{\omega_{3}-\omega_{4}}+\right. \\
& \left.+\left(\frac{\sigma-1}{\sigma}\right)^{\sigma} \phi^{\sigma} \tau^{\sigma-1}\left(\Psi_{\widehat{n}}(g)\right)^{1-\omega_{4}}\left(\Psi_{\widehat{q}}(g)\right)^{1-\sigma} \Psi_{\widehat{I}}(g)\right]
\end{aligned}
$$

At this point, combining equations (37) and (38) it is possible to obtain explicitely the value of $g$ :

$$
g=[\rho(1+\varepsilon)]^{\frac{1}{\omega_{4}}}
$$

(as already found in the analysis of the balanced growth path), and therefore all the long run levels can be expressed in terms of the parameters $\alpha, \lambda, \sigma$, $\rho, \varepsilon$. 
In conclusion, the results obtained are summarized in the following Proposition:

Proposition 3 In the model considered with human capital accumulation, there exist explicit functions expressing the long run levels $\widehat{q}, \widehat{C}, \widehat{I}, \widehat{K}, \widehat{H}, \widehat{n}$, $r, u$ exclusively in terms of $g$ :

$$
\begin{array}{llll}
\widehat{q}=\Psi_{\widehat{q}}(g) & \widehat{C}=\Psi_{\widehat{C}}(g) & \widehat{I}=\Psi_{\widehat{I}}(g) & \widehat{K}=\Psi_{\widehat{K}}(g) \\
\widehat{H}=\Psi_{\widehat{H}}(g) & \widehat{n}=\Psi_{\widehat{n}}(g) & r=\Psi_{r}(g) & u=\Psi_{u}(g)
\end{array}
$$

with:

$$
g=[\rho(1+\varepsilon)]^{\frac{1}{\omega_{4}}}
$$

From the analysis of the expressions $\Psi_{()}(g)$ it is possible to deduce the effects of the different exogenous variables on the long run levels in the economy. In particular, it turns out that the function $\Psi_{\widehat{q}}(g)$ depends on $z, e$ and $\varepsilon$ and the functions $\Psi_{\widehat{n}}(g), \Psi_{\widehat{I}}(g), \Psi_{\widehat{K}}(g), \Psi_{\widehat{C}}(g)$ and $\Psi_{\widehat{H}}(g)$ depend on $z, e$, $\tau$ and $\varepsilon$ (the productivity variables in the different sectors). In addition, the long run growth factor $g$ is not affected by the productivity of the final good sector, of the equipment sector and of the intermediate good sector, while it is affected by the productivity of schooling, as expressed in the following Proposition:

Proposition 4 Assuming that a solution for the steady state system exists, the long run values of $z$, e and $\varepsilon$ affect the stationary values $\widehat{q}, \widehat{n}, \widehat{I}, \widehat{K}, \widehat{C}$ and $\widehat{H}$ and the long run value of $\tau$ affects the stationary values $\widehat{n}, \widehat{I}, \widehat{K}, \widehat{C}$ and $\widehat{H}$. Furthermore $z$, e and $\tau$ have no impact on the long term growth factor $g$, while $\varepsilon$ affects it.

In conclusion, therefore, in this version of the model the true engine of growth is represented by the accumulation of human capital, through the schooling activity. Finally, in this case it is also possible to compute the derivative $\frac{d g}{d \sigma}$, that is given by:

$$
\frac{d g}{d \sigma}=[\rho(1+\varepsilon)]^{\frac{1}{\omega_{4}}} \cdot \log [\rho(1+\varepsilon)] \cdot\left(-\frac{\lambda \alpha(1-\alpha+\lambda \alpha)}{[\sigma(1-\alpha+\lambda \alpha)-1+\alpha-2 \lambda \alpha]^{2}}\right)
$$

and is negative (since the first two factors are positive - in particular $\log [\rho(1+\varepsilon)]>0$ because $\rho>\frac{1}{1+\varepsilon}$ and hence $\rho(1+\varepsilon)>1$ - while the 
third factor is negative). In this model there is therefore a negative relation between the elasticity of substitution (that is also a measure of competitivity) and the growth rate of the economy, and an increase in the monopoly power (i.e. a reduction in $\sigma$ ) increases growth. Other papers, starting from the assumption that the engine of growth is the continuous improvement of the quality level of the existing goods, conclude that product market competition is unambiguously good for growth. The present study shows that this is no longer true when we consider an horizontal differentiation model of endogenous growth where firms invent new varieties of intermediate goods but, at the same time, the true engine of growth is represented by human capital accumulation.

\section{Simulation of the model}

The model described above can then be simulated numerically in order to study a number of issues of interest, concerning in particular the short run behavior of the economy. First of all, this requires a calibration, and the values of the different parameters are chosen in such a way that the model is able to reproduce the most relevant empirical data that are available (with reference to this aspect see also the previous work, Mattalia 2002). In particular, the values chosen for the different parameters are reported in the following table:

\begin{tabular}{|l|l|l|}
\hline Parameter & Symbol & Value \\
\hline Capital share in the final good sector & $\alpha$ & 0.35 \\
\hline Share of software in the production of efficient capital & $\lambda$ & 0.85 \\
\hline Elasticity of substitution between varieties of sofwares & $\sigma$ & 1.40 \\
\hline Rate of depreciation of physical capital & $\delta$ & 0.10 \\
\hline Psychological discount factor & $\rho$ & 0.96 \\
\hline Total factor productivity in the final good sector & $z$ & 3 \\
\hline Total factor productivity in the equipment sector & $e$ & 12 \\
\hline Human capital productivity in the intermediate good sector & $\tau$ & 0.25 \\
\hline Productivity in the education sector & $\varepsilon$ & 0.044 \\
\hline Cost of a new variety of software in units of output & $\eta$ & 20 \\
\hline
\end{tabular}

and with these parameters the steady state of the model leads to the following 
values for some relevant quantities:

\begin{tabular}{|l|l|}
\hline Capital/output ratio & 2.5 \\
\hline R\&D expenditure in terms of GDP & $3.5 \%$ \\
\hline Interest rate & $5 \%$ \\
\hline Growth rate of output & $1 \%$ \\
\hline
\end{tabular}

Starting from this benchmark situation, different types of simulations are considered. In particular, first of all it is possible to study how the economy reacts to shocks on the productivities of the different sectors (and to compare the results with those of the model presented in the previous work, where human capital accumulation was not present). A second aspect of interest is the link between competitivity and growth; since analytically it has been obtained an inverse relation in the long run between these two quantities, it is important to study how this relation works in the short run and which are the mechanisms that explain it. A third result is that it is possible to show the presence of "imbalance effects" with reference to some variables (in particular the ratio between physical and human capital, the ratio between physical capital and $R \& D$ expenditure and the ratio between human capital and $R \& D$ expenditure): when the initial value of these variables is different from the steady state value (in particular, lower) the growth rate of the economy is higher than the rate we have in correspondence of the steady state value of the variables considered. Finally, it is possible to consider some extensions of the model, in particular introducing the notion of "broad output", different types of subsidies (on R\&D, on the accumulation of physical capital, on the accumulation of human capital) and a situation in which the productivity of human capital is a function of technological progress. The results in these cases are that imbalance effects are still present and that, contrary to other types of subsidies, the introduction of subsidies on human capital accumulation has (in the short run) a positive effect on growth.

\subsection{Productivity shocks}

The first aspect considered is represented by the effects of different shocks in the productivity variables; all these shocks are permanent (from $t=0$ ) and have an intensity of $1 \%$.

The first situation is an increase in $z$ (the productivity of the final good sector). From the analytical results derived above we know that this increase 
has no effect on long term growth, and in fact in the long run the growth rates of output, physical capital and human capital don't change with respect to the initial situation. This is a central difference with respect to the model (presented in the previous work) without human capital accumulation, where an increase in $z$ increases long term growth. The reason is that, like in that model, the lab-equipment specification implies that the production function in the R\&D sector is the same as in the final good sector, so that an increase in the productivity of the latter is equivalent to an increase in the productivity of R\&D; nevertheless, this is no longer the engine of growth of the model (that is now represented by the accumulation of human capital), and hence an increase in its productivity doesn't have long run effects on growth.

With reference to the short run, there is initially an increase in the human capital fraction employed in education and a consequent reduction in the fraction employed in production (where the increase in the productivity of the final good sector reduces the cost of production of the final good and determines a reallocation of human capital favorable to the final good sector and at the expenses of the intermediate good sector). As a consequence, initially there is an increase in the growth rate of human capital (because of the increase in the human capital fraction employed in education) and a decrease in the growth rate of output (because human capital accumulation influences growth with a lag, since education requires time before producing its effects on the economy). After some time, then, the situation reverses, the human capital fraction in education and the consequent growth of human capital reduce, while the growth rate of output increases (because the previous accumulation of human capital produces its effects) and returns to its initial level.

The same type of dynamics arise when we consider, instead of an increase in $z$, a decrease in $\eta$ (the cost of a new variety of software). Indeed, a reduction in this cost (that is expressed in terms of output, as a consequence of the lab-equipment assumption) is analogous to an increase in the productivity of the final good sector, and this explains why the results obtained are essentially of the same kind.

The second situation analyzed is an increase in $e$ (the productivity of the equipment sector). Also this kind of shock has no long run effect, because an increase in the productivity of the equipment sector increases the production of efficient capital and therefore of the final good, that corresponds (as a consequence of the lab-equipment assumption) to an expansion in the $\mathrm{R} \& \mathrm{D}$ 
sector, but since this is no longer the engine of growth of the model there is no effect on long term growth.

With reference to the short run behavior of the different quantities, in this case initially there is an increase in the human capital fraction employed in education and a consequent decrease in the fraction employed in production (where the increase in the profitability of producing efficient capital and of the production of sofwares determines a rise in the human capital fraction employed in the intermediate good sector, at the expenses of the fraction employed in the final good sector). In this situation the growth of output initially slightly decreases and then increases, then the situation partially reverses, the human capital fraction in education decreases and the growth rate of output returns to its initial level.

The same kind of dynamics can be obtained considering an increase in $\tau$ (the productivity of the intermediate good sector). In fact, this rise reduces the cost of softwares and increases their production, originating the same type of behavior observed in the case of an increase in $e$.

The third situation studied is an increase in $\varepsilon$ (the productivity of schooling). In this case, contrary to the previous kind of shocks, there are long run effects on growth, since the accumulation of human capital through education is the true engine of growth of this model. In particular, the rise in the productivity of schooling initially determines a decrease in the human capital fraction employed in education and a consequent increase in the fraction employed in production (where the fraction of human capital in the final good sector increases, at the expenses of the fraction employed in the intermediate good sector). This originates an increase in the growth rate of output (initially very strong, and that then partially reduces), so that the final result is a permanent positive effect on the growth of the economy (more precisely, as a consequence of a $1 \%$ increase in the productivity of schooling the growth rate of output increases from $1.04 \%$ to $1.24 \%$ ).

\subsection{Competitivity and growth}

The second aspect considered trough the simulations is represented by the link between competitivity and growth as a consequence of the presence of human capital accumulation. This can be analyzed examining the effects of an increase in $\sigma$, that is the elasticity of substitution between varieties of softwares and that can be seen as a measure of competitivity in the economy. 
In this case there is a long run effect on the economy, represented (as shown analytically) by a reduction in the growth rate of output. More precisely, the increase in $\sigma$ initially determines a reduction in the human capital fraction employed in education and a consequent rise in the fraction employed in production, where in particular there is a reallocation of human capital from the final good sector to the intermediate good sector. The intuition is that an increase in the degree of competition of the intermediate sector increases the output of this sector and its demand of human capital. At the same time a reduction in the mark-up rate (that is inversely related to $\sigma$ ) makes it more profitable for the final good producers to substitute capital goods for human capital, and this causes the reallocation of human capital between these two sectors. These movements, together with the reduction in the human capital employed in education, determine the fall in the growth of output with respect to the initial situation (more precisely, as a consequence of a $1 \%$ increase in the elasticity of substitution between varieties of softwares the growth rate of output decreases from $1.04 \%$ to $0.93 \%$ ). On the contrary, a reduction in $\sigma$ (and hence an increase in the monopoly power), has a positive effect on the growth of the economy.

As observed above, this is an important difference with respect to other studies that assume the continuous improvement of the quality level of goods as the engine of growth, and conclude that product market competition has a positive effect on the economy. In the present analysis we have that this is no longer true if we consider a model of endogenous growth with horizontal differentiation, where firms invent new varieties of intermediate goods but, at the same time, the engine of growth is represented by human capital accumulation. In this case, in fact, the relation between competition and growth reverses.

\subsection{Imbalance effects}

The third aspect considered is the study of "imbalance effects" with reference to some variables. The typical imbalance emerges considering the relationship between the growth rate of output and the ratio $\frac{K}{H}$ (physical capital/human capital), and a deep analysis of this topic in endogenous growth models is presented in Barro and Sala-i-Martin (1995). The central idea is that when the value of the ratio $\frac{K}{H}$ is different from the steady state value (in particular, when it is lower), the growth rate of the economy is higher than the rate we have in correspondence of the steady state value of $\frac{K}{H}$. 
Considering first of all a one-sector model in which physical and human capital are produced by identical production functions, this setup implies a particular steady state value for the ratio $\frac{K}{H}$, and if the initial value of this ratio is different from the steady state one there are adjustments in the two stocks until the steady state value is attained. In this case, imposing the restriction according to which gross investment in physical and human capital cannot be negative (because this would not be realistic) it is possible to obtain interesting results concerning the effects on growth due to imbalances between the levels of physical and human capital.

In particular, if the economy starts from a situation in which human capital $H$ is initially abundant relative to physical capital $K$, so that the ratio $\frac{K}{H}$ is below its steady state value, then the agents realize that they have too much $H$ in relation to $K$, and since it is not feasible to have negative gross investment in $H$, they don't invest in $H$ and they allow $H$ to depreciate. At this point the growth rates of $K$ and $Y$ decline monotonically over time (as in the standard neoclassical growth model), and since $H$ declines (at the depreciation rate) and $K$ increases (at a decreasing rate) the ratio $\frac{K}{H}$ rises over time. These results imply that the growth rate of output is inversely related to the ratio $\frac{K}{H}$ when this ratio is below its steady state value, and this relation is called "imbalance effect", since the greater the imbalance (that is the further $\frac{K}{H}$ is below its steady state value) the higher the growth rate. The results are analogous if the economy begins with a relative abundance of physical capital, so that the ratio $\frac{K}{H}$ is above its steady state value, and in this case the agents don't invest in $K$ and they allow $K$ to depreciate. The setup is again equivalent to the standard neoclassical growth model, therefore the growth rates of $H$ and $Y$ decline monotonically over time, and since $K$ declines (at the depreciation rate) and $H$ increases (at a decreasing rate) the ratio $\frac{K}{H}$ falls over time. These results imply that the growth rate of output is positively related to the ratio $\frac{K}{H}$ when this ratio is above its steady state value, therefore there is again an "imbalance effect", since the greater the imbalance (that is the further $\frac{K}{H}$ is above its steady state value) the higher the growth rate.

The result of this analysis is that the relation between the growth rate of output and the ratio $\frac{K}{H}$ follows a U-shaped function; the minimal growth rate corresponds to the steady state ratio of $\frac{K}{H}$, and on either side of the steady state the growth rate rises symmetrically with the magnitude of the gap between $\frac{K}{H}$ and its steady state value. According to the theory, therefore, 
a reduction of physical capital (Barro and Sala-i-Martin give the example of a war that destroys $K$ but not $H$ ) has the same effect on the growth rate of a reduction of human capital (due for instance to an epidemic that destroys $H$ but not $K)$.

In reality, it is most plausible that an increase in $\frac{K}{H}$ above its steady state value has only a small positive effect on the growth rate, lower than the corresponding effect due to a decrease in $\frac{K}{H}$ below its steady state value, and therefore it is possible to consider an extension of the theory that leads to asymmetric effects from $\frac{K}{H}$ below or above its steady state value. In particular, it is possible to introduce adjustment costs for capital accumulation, that can be assumed to be greater for $H$ than for $K$; in this case a relative abundance of $H$ would lead to substantial investment in $K$ and, as a consequence, to a high growth rate of output, but a relative abundance of $K$ would have a much smaller effect on investment in $H$ and, hence, on the growth rate of output. If this is true, the relation between the growth rate of output and the ratio $\frac{K}{H}$ still follows a U-shaped function, but (since the adjustment costs for changing human capital are greater than those for changing physical capital) the sensitivity of the growth rate to $\frac{K}{H}$ is larger when this ratio is below its steady state value than when it is above its steady state value. This model therefore predicts that an economy would recover much faster from a war that destroyed mainly $K$ than from an epidemic that destroyed mainly $H$.

The same result can be obtained considering a two-sector model in which physical and human capital are produced by different technologies, assuming also that education (i.e. the production of new human capital) is relatively intensive in human capital as an input (because the assumption made in the first model that physical goods and education are generated by the same production functions neglected a key aspect of education, i.e. the fact that it relies heavily on educated people as an input). This modification of the production structure creates an asymmetry in the effects on the growth rate that derive from imbalances between physical and human capital, so that the growth rate rises with one type of imbalance and falls with the other type in the neighborhood of the steady state. In particular, the result is that the growth rate of output still increases with the magnitude of the imbalance between $K$ and $H$ if human capital is relatively abundant, but tends to fall (or to increase more slowly) with the magnitude of the imbalance if human capital is relatively scarce. This result is due to the assumption that the education sector is relatively intensive in human capital; in this case, if the 
ratio $\frac{K}{H}$ is higher than its steady state value, then the marginal product of human capital in the goods sector is high, and growth occurs mainly because of the high growth rate of human capital (indeed this rate is increasing in $\left.\frac{K}{H}\right)$. The high level of $\frac{K}{H}$ implies however a high wage rate and therefore a high cost of operation for the sector of education which is relatively intensive in human capital. This motivates people to allocate human capital to the production of goods rather than to education and this effect tends to retard the growth rate of the economy.

In conclusion, considering a one-sector model in which physical and human capital are produced by the same technology, the growth rate of output rises with the magnitude of the gap between the ratio $\frac{K}{H}$ and its steady state value, generating an "imbalance effect" that is symmetric (i.e. higher growth rates of output emerge if either $K$ or $H$ is in relatively short supply), so that shortfalls of human capital will have approximately the same effects on growth as shortfalls of physical capital (which is not realistic). Considering a more realistic two-sector model in which physical and human capital are produced by different technologies (with the production of human capital that is relatively intensive in human capital), the conclusions about the imbalance effect change, since the growth rate of output now tends to rise with the magnitude of the imbalance between the ratio $\frac{K}{H}$ and its steady state value if human capital is relatively abundant, but tends to decline (or to rise more slowly) with the magnitude of the imbalance if human capital is relatively scarce.

The model presented in this paper is a multi-sectoral model with human capital, hence these latter conclusions should apply to it. To verify this point, it is possible to use the data obtained from the simulations presented above to derive, first of all, the relationship between the growth factor of output $g_{Y}$ and the ratio between physical and human capital $\frac{K}{H}$; the results are reported in the following graphic:

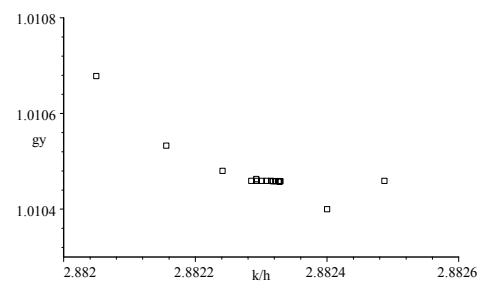


From this picture the presence of an imbalance effect is confirmed, and it turns out that (as the theory predicts) the growth factor (and therefore the growth rate) of output rises with the magnitude of the imbalance when the ratio $\frac{K}{H}$ is below its steady state value (here this steady state value is 2.8824 , that corresponds to the minimal growth rate of output), while it rises more slowly with the magnitude of the imbalance when the ratio $\frac{K}{H}$ is above its steady state value.

A similar result holds with reference to the relation between the growth factor of output $g_{Y}$ and the ratio between physical capital and R\&D expenditure $\frac{K}{R D}$, in fact in this case we have:

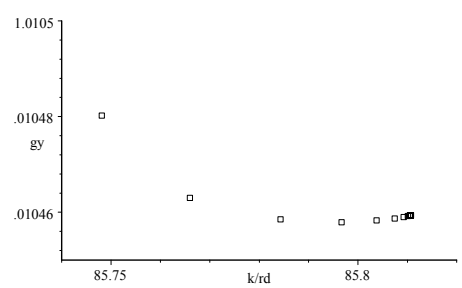

and again there is an asymmetric imbalance effect, with the growth rate of output that rises with the magnitude of the imbalance only when the ratio $\frac{K}{R D}$ is lower than its steady state value.

The same kind of result, finally, can be obtained considering the relation between the growth factor of output $g_{Y}$ and the ratio between human capital and $R \& D$ expenditure $\frac{H}{R D}$, in fact in this situation we obtain:

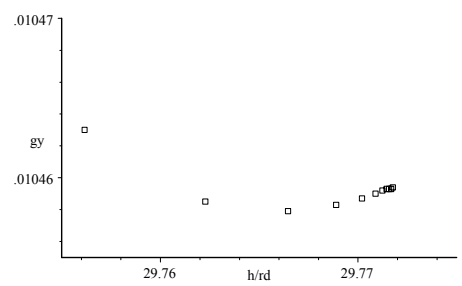

and also in this case there is an (asymmetric) imbalance effect.

These results have some implications, for instance considering the situation of underdeveloped countries. These countries are characterized by the presence of low levels of human capital and of R\&D expenditures, hence the ratios $\frac{K}{H}$ and $\frac{K}{R D}$ are high. According to the results obtained, the corresponding growth rates of output are lower than the values that would prevail if the 
same ratios were smaller, hence these countries will grow at lower rates than countries starting from higher levels of human capital and of R\&D expenditures, and they will not be able to reach the latter.

\section{Extensions of the model}

\subsection{The notion of broad output}

The last aspect of the analysis consists in considering some extensions of the model introduced above, in order to verify how the basic results obtained change as a consequence of these modifications. The first extension is represented by the introduction of the notion of "broad output", a concept used by Barro and Sala-i-Martin (1995) in their analysis of imbalance effects. Indeed, measured output can be broadened to include gross investment in human capital multiplied by an appropriate shadow price of human capital. Broad output is therefore defined as the sum of narrow output and the value in units of goods of the gross investment in human capital, i.e.:

$$
Q_{t}=Y_{t}+w_{t}\left(H_{t}-H_{t-1}\right)
$$

where $Q_{t}$ is broad output, $Y_{t}$ is narrow output, $w_{t}$ represents the price of human capital in terms of output and $\left(H_{t}-H_{t-1}\right)$ is the investment in human capital. This represents a new equilibrium equation of the model, and rewriting it along a balanced growth path we obtain the following further restriction that must hold among the various growth factors:

$$
g_{Q}=g_{Y}=g_{w} g_{H}
$$

where $g_{Q}$ represents the long-run growth factor of broad output, that therefore equals the long-run growth factor of narrow output.

It is then possible to introduce the stationarized dynamic equation corresponding to the original equilibrium equation written above, getting:

$$
\widehat{Q}_{t}=\widehat{Y}_{t}+\widehat{n}_{t}^{\omega_{4}} \widehat{H}_{t}\left(1-\frac{1}{g_{H}}\right)
$$

where $\widehat{Y}_{t}=\frac{Y_{t}}{w_{t}^{\omega_{2}}}$ and $\widehat{Q}_{t}=\frac{Q_{t}}{w_{t}^{\omega_{2}}}$. In the same way the stationarized steady state equation corresponding to the original one can be obtained, and is given by:

$$
\widehat{Q}=\widehat{Y}+\widehat{n}^{\omega_{4}} \widehat{H}\left(1-\frac{1}{g_{H}}\right)
$$


where $\widehat{Y}=\frac{\bar{Y}}{\bar{w}^{\omega_{2}}}$ and $\widehat{Q}=\frac{\bar{Q}}{\bar{w}^{\omega_{2}}}$. At this point it is possible to perform the simulations of the model considering also the new equilibrium equation, and the results of these simulations can be used to derive the relationship between the growth factor of broad output $g_{Q}$ and the ratio between physical and human capital $\frac{K}{H}$; the results are reported in the following graphic:

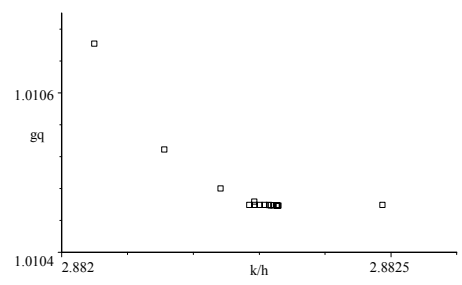

From this picture we have that the growth factor of broad output tends to be a monotonically decreasing function of the ratio between physical and human capital (exactly as obtained by Barro and Sala-i-Martin, 1995). When the ratio $\frac{K}{H}$ is below its steady state value the growth rate of broad output rises with the magnitude of the imbalance, while when this ratio is above its steady state value the growth rate of broad output declines with the magnitude of the imbalance. Also when we introduce the notion of broad output, therefore, an asymmetric imbalance effect is present in the model.

\subsection{The introduction of subsidies}

A second extension of the model that can be considered is the introduction of different types of subsidies. In particular, it is possible to consider subsidies on R\&D, on physical capital accumulation or on human capital accumulation.

The first possibility is the introduction of a subsidy on $R \& D$, so that the cost of research becomes $\eta\left(1-\beta_{R, t}\right)$ where $\beta_{R, t}$ is the subsidy rate on $\mathrm{R} \& \mathrm{D}$. In this case the equations that change in the basic structure of the model are the free-entry condition, that becomes:

$$
\eta\left(1-\beta_{R, t}\right)=\sum_{z=t}^{\infty} R_{t}^{z} \frac{1}{\sigma-1} \frac{w_{z}}{\tau_{z}} x_{i, z}
$$

and the equilibrium on the final good market, that becomes:

$$
Y_{t}=C_{t}+I_{t}+\eta\left(1-\beta_{R, t}\right) \triangle n_{t}
$$


As a consequence, the equilibrium equations (16) and (23) are substituted by the equations:

$$
\begin{gathered}
z_{t}^{\frac{1}{\alpha}} K_{t}\left(\frac{1-\alpha}{w_{t}}\right)^{\frac{1-\alpha}{\alpha}}=C_{t}+I_{t}+\eta\left(1-\beta_{R, t}\right) \triangle n_{t} \\
\frac{\tau_{t}^{1-\sigma}(\sigma-1)^{1-\sigma} \sigma^{\sigma}}{\phi^{\sigma}} \cdot \frac{\eta\left(1-\beta_{R, t}\right) r_{t+1}}{1+r_{t+1}}=w_{t}^{1-\sigma} q_{t}^{1-\sigma} I_{t}
\end{gathered}
$$

Rewriting these equations along a balanced growth path it is possible to obtain the corresponding restrictions that must hold for the growth factors, and that are:

$$
\begin{gathered}
g_{C}=g_{I}=g_{n}=g_{K}\left(g_{w}\right)^{-\frac{1-\alpha}{\alpha}} \\
\left(g_{w}\right)^{1-\sigma}\left(g_{q}\right)^{1-\sigma} g_{I}=1
\end{gathered}
$$

i.e. they are the same restrictions (expressed by equations (25) and (32) above) that hold in absence of subsidies on R\&D. The presence of these subsidies, therefore, doesn't have long run effects.

The equations of the stazionarized dynamic system that change are the second and the last one, that now become:

$$
\begin{gathered}
z_{t}^{\frac{1}{\alpha}} \widehat{K}_{t} \widehat{n}_{t}^{\omega_{3}}(1-\alpha)^{\frac{1-\alpha}{\alpha}}=\widehat{C}_{t}+\widehat{I}_{t}+\eta\left(1-\beta_{R, t}\right) \widehat{n}_{t}\left(1-\frac{1}{g_{t}}\right) \\
\frac{\tau_{t}^{1-\sigma}(\sigma-1)^{1-\sigma} \sigma^{\sigma}}{\phi^{\sigma}} \cdot \frac{\eta\left(1-\beta_{R, t}\right) r_{t+1}}{1+r_{t+1}}=\widehat{q}_{t}^{1-\sigma} \widehat{I}_{t}
\end{gathered}
$$

and, similarly, the equations of the stationarized steady state system that change are equations (34) and (41), that are substituted by the equations:

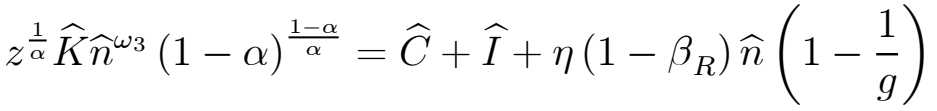

$$
\begin{aligned}
& \frac{\tau^{1-\sigma}(\sigma-1)^{1-\sigma} \sigma^{\sigma}}{\phi^{\sigma}} \cdot \frac{\eta\left(1-\beta_{R}\right) r}{1+r}=\widehat{q}^{1-\sigma} \widehat{I}
\end{aligned}
$$

Finally, the long run levels that change are $\widehat{I}$ and $\widehat{C}$, that now become:

$$
\widehat{I}=\Psi_{\widehat{I}}(g)=\frac{\tau^{1-\sigma}(\sigma-1)^{1-\sigma} \sigma^{\sigma}}{\phi^{\sigma}} \cdot \frac{\eta\left(1-\beta_{R}\right) \Psi_{r}(g)}{1+\Psi_{r}(g)} \cdot \frac{1}{\left(\Psi_{\widehat{q}}(g)\right)^{1-\sigma}}
$$


$\widehat{C}=\Psi_{\widehat{C}}(g)=z^{\frac{1}{\alpha}} \Psi_{\widehat{K}}(g)\left(\Psi_{\widehat{n}}(g)\right)^{\omega_{3}}(1-\alpha)^{\frac{1-\alpha}{\alpha}}-\Psi_{\widehat{I}}(g)-\eta\left(1-\beta_{R}\right)\left(1-\frac{1}{g}\right) \Psi_{\widehat{n}}(g)$

These expressions can be used to perform new simulations, and the main result that can be obtained from these simulations is that, also in the short run, the growth rates of the various quantities don't change with respect to the basic model considered above. In conclusion, therefore, the introduction of subsidies on R\&D doesn't have significative effects on growth, neither in the long run nor in the short run.

The second possibility is the introduction of a subsidy on physical capital accumulation, so that the price of physical capital becomes $d_{t}\left(1-\beta_{K, t}\right)$ where $\beta_{K, t}$ is the subsidy rate on physical capital accumulation. In this case the only equation that changes in the basic structure of the model is the one that gives the profit of the final good producer, that becomes:

$$
\pi_{t}=\sum_{s=t}^{\infty}\left[Y_{s}-w_{s} H_{Y, s}\right] R_{t}^{s}-d_{t}\left(1-\beta_{K, t}\right) E_{t}
$$

but then the price of physical capital doesn't appear explicitely in the equilibrium conditions, that therefore don't change with respect to the basic model. In conclusion, therefore, also a subsidy on physical capital accumulation doesn't have any effect on growth, neither in the long run nor in the short run.

The third possibility is the introduction of a subsidy on human capital accumulation, so that the price of human capital becomes $w_{t}\left(1-\beta_{H, t}\right)$ where $\beta_{H, t}$ is the subsidy rate on human capital accumulation. In this case the equations that change in the basic structure of the model are the demand of human capital by the final good sector, that becomes:

$$
H_{Y, t}=\left(\frac{(1-\alpha) z_{t}}{w_{t}\left(1-\beta_{H, t}\right)}\right)^{\frac{1}{\alpha}} K_{t}
$$

the price of intermediate input $i$, that becomes:

$$
p_{i, t}=\left(\frac{\sigma}{\sigma-1}\right) \frac{w_{t}\left(1-\beta_{H, t}\right)}{\tau_{t}} \quad \forall i \in\left[0, n_{t}\right]
$$


and the free-entry condition, that becomes:

$$
\eta=\sum_{z=t}^{\infty} R_{t}^{z} \frac{1}{\sigma-1} \frac{w_{z}\left(1-\beta_{H, z}\right)}{\tau_{z}} x_{i, z}
$$

As a consequence, the equilibrium conditions expressed by equations (15), (16), (17), (22) and (23) are substituted by the following equations:

$$
\begin{aligned}
&\left(\frac{(1-\alpha) z_{t}}{w_{t}\left(1-\beta_{H, t}\right)}\right)^{\frac{1}{\alpha}} K_{t}+n_{t}\left(\frac{\sigma-1}{\sigma}\right)^{\sigma}\left(\frac{\phi}{w_{t}\left(1-\beta_{H, t}\right)}\right)^{\sigma} q_{t}^{1-\sigma} I_{t} \tau_{t}^{\sigma-1}=u_{t} H_{t-1} \\
& z_{t}^{\frac{1}{\alpha}} K_{t}\left(\frac{1-\alpha}{w_{t}\left(1-\beta_{H, t}\right)}\right)^{\frac{1-\alpha}{\alpha}}=C_{t}+I_{t}+\eta \Delta n_{t} \\
& \alpha z_{t}^{\frac{1}{\alpha}}(1-\lambda) e_{t} q_{t}^{\lambda}\left(\frac{1-\alpha}{w_{t}\left(1-\beta_{H, t}\right)}\right)^{\frac{1-\alpha}{\alpha}}=1-\left(\frac{1-\delta}{1+r_{t+1}}\right)\left(\frac{e_{t}}{e_{t+1}}\right)\left(\frac{q_{t}}{q_{t+1}}\right)^{\lambda} \\
& \frac{w_{t}\left(1-\beta_{H, t}\right) q_{t}}{\tau_{t} \phi}=n_{t}^{\frac{1}{\sigma-1}}\left(\frac{\sigma-1}{\sigma}\right) \\
& \frac{\tau_{t}^{1-\sigma}(\sigma-1)^{1-\sigma} \sigma^{\sigma}}{\phi^{\sigma}} \cdot \frac{\eta r_{t+1}}{1+r_{t+1}}=\left(w_{t}\left(1-\beta_{H, t}\right)\right)^{1-\sigma} q_{t}^{1-\sigma} I_{t}
\end{aligned}
$$

Rewriting these equations along a balanced growth path we get the restrictions that must hold for the various growth factors; these relations are the same that hold in absence of subsidies on human capital accumulation, hence it is possible to conclude that the presence of these subsidies doesn't have long run effects.

At this point, the equations of the stationarized dynamic system that change are the first three and the last two, that are replaced by:

$$
\begin{gathered}
\left(\frac{(1-\alpha) z_{t}}{1-\beta_{H, t}}\right)^{\frac{1}{\alpha}} \widehat{K}_{t} \widehat{n}_{t}^{\omega_{3}-\omega_{4}}+\left(\frac{\sigma-1}{\sigma}\right)^{\sigma}\left(\frac{\phi}{1-\beta_{H, t}}\right)^{\sigma} \tau_{t}^{\sigma-1} \widehat{n}_{t}^{1-\omega_{4}} \widehat{q}_{t}^{1-\sigma} \widehat{I}_{t}=u_{t} \widehat{H}_{t-1} g_{t}^{-\omega_{4}} \\
z_{t}^{\frac{1}{\alpha}} \widehat{K}_{t} \widehat{n}_{t}^{\omega_{3}}\left(\frac{1-\alpha}{1-\beta_{H, t}}\right)^{\frac{1-\alpha}{\alpha}}=\widehat{C}_{t}+\widehat{I}_{t}+\eta \widehat{n}_{t}\left(1-\frac{1}{g_{t}}\right)
\end{gathered}
$$




$$
\begin{gathered}
\alpha z_{t}^{\frac{1}{\alpha}}(1-\lambda) e_{t} \widehat{q}_{t}^{\lambda}\left(\frac{1-\alpha}{1-\beta_{H, t}}\right)^{\frac{1-\alpha}{\alpha}}+\frac{1-\delta}{1+r_{t+1}}\left(\frac{e_{t}}{e_{t+1}}\right)\left(\frac{\widehat{q}_{t}}{\widehat{q}_{t+1}}\right)^{\lambda}\left(\frac{\widehat{n}_{t+1}}{\widehat{n}_{t} g_{t+1}}\right)^{\omega_{3}-1}=1 \\
\frac{\left(1-\beta_{H, t}\right) \widehat{q}_{t}}{\tau_{t} \phi}=\widehat{n}_{t}^{\frac{1}{\sigma-1}}\left(\frac{\sigma-1}{\sigma}\right) \\
\frac{\tau_{t}^{1-\sigma}(\sigma-1)^{1-\sigma} \sigma^{\sigma}}{\phi^{\sigma}} \cdot \frac{\eta r_{t+1}}{1+r_{t+1}}=\left(1-\beta_{H, t}\right)^{1-\sigma} \widehat{q}_{t}^{1-\sigma} \widehat{I}_{t}
\end{gathered}
$$

Similarly, the equations of the stationarized steady state system that change are equations (33), (34), (35), (40) and (41) that are substituted by the equations:

$$
\begin{aligned}
& \left(\frac{(1-\alpha) z}{1-\beta_{H}}\right)^{\frac{1}{\alpha}} \widehat{K} \widehat{n}^{\omega_{3}-\omega_{4}}+\left(\frac{\sigma-1}{\sigma}\right)^{\sigma}\left(\frac{\phi}{1-\beta_{H}}\right)^{\sigma} \tau^{\sigma-1} \widehat{n}^{1-\omega_{4}} \widehat{q}^{1-\sigma} \widehat{I}=u \widehat{H} g^{-\omega_{4}} \\
& z^{\frac{1}{\alpha}} \widehat{K}^{\omega^{\omega}}\left(\frac{1-\alpha}{1-\beta_{H}}\right)^{\frac{1-\alpha}{\alpha}}=\widehat{C}+\widehat{I}+\eta \widehat{n}\left(1-\frac{1}{g}\right) \\
& \alpha z^{\frac{1}{\alpha}}(1-\lambda) e \widehat{q}^{\lambda}\left(\frac{1-\alpha}{1-\beta_{H}}\right)^{\frac{1-\alpha}{\alpha}}+\frac{1-\delta}{1+r} g^{1-\omega_{3}}=1 \\
& \tau \phi\left(\frac{\sigma-1}{\sigma}\right) \widehat{n}^{\frac{1}{\sigma-1}}=\left(1-\beta_{H}\right) \widehat{q} \\
& \frac{\tau^{1-\sigma}(\sigma-1)^{1-\sigma} \sigma^{\sigma}}{\phi^{\sigma}} \cdot \frac{\eta r}{1+r}=\left(1-\beta_{H}\right)^{1-\sigma} \widehat{q}^{1-\sigma} \widehat{I}
\end{aligned}
$$

Finally, the long run levels that change are:

$$
\begin{gathered}
\widehat{q}=\Psi_{\widehat{q}}(g)=\left(\frac{1-\frac{1-\delta}{1+\Psi_{r}(g)} g^{1-\omega_{3}}}{\alpha z^{\frac{1}{\alpha}}(1-\lambda) e\left(\frac{1-\alpha}{1-\beta_{H}}\right)^{\frac{1-\alpha}{\alpha}}}\right)^{\frac{1}{\lambda}} \\
\widehat{n}=\Psi_{\widehat{n}}(g)=\left(\frac{\tau \phi}{\left(1-\beta_{H}\right) \Psi_{\widehat{q}}(g)} \cdot \frac{\sigma-1}{\sigma}\right)^{1-\sigma} \\
\widehat{I}=\Psi_{\widehat{I}}(g)=\frac{\tau^{1-\sigma}(\sigma-1)^{1-\sigma} \sigma^{\sigma}}{\phi^{\sigma}} \cdot \frac{\eta \Psi_{r}(g)}{1+\Psi_{r}(g)} \cdot \frac{1}{\left(1-\beta_{H}\right)^{1-\sigma}\left(\Psi_{\widehat{q}}(g)\right)^{1-\sigma}}
\end{gathered}
$$




$$
\begin{aligned}
\widehat{C}=\Psi_{\widehat{C}}(g)=z^{\frac{1}{\alpha}} \Psi_{\widehat{K}}(g)\left(\Psi_{\widehat{n}}(g)\right)^{\omega_{3}}\left(\frac{1-\alpha}{1-\beta_{H}}\right)^{\frac{1-\alpha}{\alpha}}-\Psi_{\widehat{I}}(g)-\eta\left(1-\frac{1}{g}\right) \Psi_{\widehat{n}}(g) \\
\widehat{H}=\Psi_{\widehat{H}}(g)=\frac{g^{\omega_{4}}}{u}\left[\left(\frac{(1-\alpha) z}{1-\beta_{H}}\right)^{\frac{1}{\alpha}} \Psi_{\widehat{K}}(g)\left(\Psi_{\widehat{n}}(g)\right)^{\omega_{3}-\omega_{4}}+\right. \\
\left.+\left(\frac{\sigma-1}{\sigma}\right)^{\sigma}\left(\frac{\phi}{1-\beta_{H}}\right)^{\sigma} \tau^{\sigma-1}\left(\Psi_{\widehat{n}}(g)\right)^{1-\omega_{4}}\left(\Psi_{\widehat{q}}(g)\right)^{1-\sigma} \Psi_{\widehat{I}}(g)\right]
\end{aligned}
$$

These expressions can be used to perform new simulations, and in this case the main result that emerges from these simulations is that, in the short run, the growth rate of output rises with respect to the basic model. In conclusion, therefore, the introduction of subsidies on human capital accumulation (that, in this model, is the true engine of growth) has a positive effect, in the short run, on the growth of the economy.

\subsection{Human capital and technological progress}

The last extension of the model that can be studied considers the productivity of human capital (i.e. of schooling) as a function of technological progress, in order to have a link between human capital accumulation and technological progress, that is typical of ICT (since new technologies can allow people to be more educated). In particular, the productivity of schooling $\varepsilon_{t}$ can be considered as a function of $g_{t}=\frac{n_{t}}{n_{t-1}}$ (the growth factor of the number of varieties of software), so that we have $\varepsilon_{t}=\varepsilon\left(g_{t}\right)$. For instance, it is possible to assume the following functional form:

$$
\varepsilon_{t}=\varepsilon\left(g_{t}\right)=a-e^{-b g_{t}} \quad a>1, b>0
$$

where $\varepsilon$ is increasing, concave and bounded.

With this new assumption, the equilibrium conditions are expressed by equations $(15)-(23)$ with the following changes in equations (18) and (20):

$$
\begin{aligned}
& H_{t}=\left[1+\left(a-e^{-b g_{t}}\right)\left(1-u_{t}\right)\right] H_{t-1} \\
& \frac{w_{t+1}}{w_{t}}=\frac{1+r_{t+1}}{1+a-e^{-b g_{t+1}}} \cdot \frac{a-e^{-b g_{t+1}}}{a-e^{-b g_{t}}}
\end{aligned}
$$


From these equations, then, we get the following restrictions that must hold along a balanced growth path (together with the other relations among the various growth factors expressed by equations $(24)-(32)$, with the exception of (27) and (29) that are replaced by the new relations):

$$
\begin{gathered}
g_{H}=\left[1+\left(a-e^{-b g_{n}}\right)(1-u)\right] \\
g_{w}=\frac{1+r}{1+a-e^{-b g_{n}}}
\end{gathered}
$$

At this point, using these restrictions it is possible to obtain the different growth factors as functions of $g_{q}$ (exactly as in the original version of the model), and from (60) we have (substituting the values found for the different variables in terms of $\left.g_{q}\right)$ :

$$
\left(g_{q}\right)^{\frac{\lambda \alpha}{1-\alpha}}=\frac{\left(g_{q}\right)^{\frac{(\sigma-1)(1-\alpha+\lambda \alpha)}{1-\alpha}}}{\rho\left(1+a-e^{-b\left(g_{q}\right)^{\frac{(\sigma-1)(1-\alpha+\lambda \alpha)}{1-\alpha}}}\right)}
$$

from which it is possible to get (in this case numerically, and not analitycally as before) the value of $g_{q}$. From (59) we then have:

$$
u=1-\frac{g_{H}-1}{a-e^{-b g_{n}}}=1-\frac{\left(g_{q}\right)^{\frac{\sigma(1-\alpha+\lambda \alpha)-1+\alpha-2 \lambda \alpha}{1-\alpha}}-1}{a-e^{-b\left(g_{q}\right)^{\frac{(\sigma-1)(1-\alpha+\lambda \alpha)}{1-\alpha}}}}
$$

and it is possible to restate Proposition 2, in which now all the growth factors and the value of $u$ are expressed in terms of $g_{q}$ (for instance $g_{Y}=\left(g_{q}\right)^{\frac{\omega_{2}}{\omega_{1}}}$ and so on), whose value is the solution of equation (61).

The stationarized dynamic system is then expressed by the same equations of the original version of the model with the following changes in the fourth and in the sixth equation:

$$
\begin{gathered}
\widehat{H}_{t} g_{t}^{\omega_{4}}=\left[1+\left(a-e^{-b g_{t}}\right)\left(1-u_{t}\right)\right] \widehat{H}_{t-1} \\
\left(\frac{\widehat{n}_{t}}{\widehat{n}_{t+1}} g_{t+1}\right)^{\frac{1}{\omega_{2}}}=\frac{1+r_{t+1}}{1+a-e^{-b g_{t+1}}} \cdot \frac{a-e^{-b g_{t+1}}}{a-e^{-b g_{t}}}
\end{gathered}
$$

and the corresponding steady state system is expressed by equations (33) - (41) with the following changes in equations (36) and (38):

$$
g^{\omega_{4}}=\left[1+\left(a-e^{-b g}\right)(1-u)\right]
$$




$$
g^{\frac{1}{\omega_{2}}}=\frac{1+r}{1+a-e^{-b g}}
$$

Proceeding as in the original version of the model the values of the different variables can now be obtained. The only differences that emerge concern the value of $u$, that from equation (62) turns out to be:

$$
u=\Psi_{u}(g)=1-\frac{g^{\omega_{4}}-1}{a-e^{-b g}}
$$

and the relation that must be satisfied by $g$, that from equations (37) and (63) is now given by:

$$
g^{\frac{1}{\omega_{2}}}=\frac{g}{\rho\left(1+a-e^{-b g}\right)}
$$

This relation allows to get (numerically, and not analytically as in the original version of the model) the value of $g$, from which all the long run levels can then be obtained. It is therefore possible to restate Proposition 3, in which all the long run levels are expressed in terms of $g$, whose value is the solution of equation (64).

With reference to this last equation, it is important to guarantee existence (and uniqueness) of its solution. In order to show this, first of all the equation can be written in the form:

$$
g^{\frac{1}{\omega_{2}}} \rho\left(1+a-e^{-b g}\right)=g
$$

At this point, to prove that the equation is satisfied for a (unique) value of $g>0$ (while it is clearly satisfied for $g=0$, that is not interesting) it is possible to consider separately the left hand side and the right hand side:

$$
\begin{aligned}
& l(g)=g^{\frac{1}{\omega_{2}}} \rho\left(1+a-e^{-b g}\right) \\
& r(g)=g
\end{aligned}
$$

The derivative of the left hand side is:

$$
l^{\prime}(g)=\frac{1}{\omega_{2}} g^{\frac{1}{\omega_{2}}-1} \rho\left(1+a-e^{-b g}\right)+g^{\frac{1}{\omega_{2}}} \rho b e^{-b g}
$$

for which we have (if $\omega_{2}>1$, so that $\frac{1}{\omega_{2}}<1$, that is the case we have when the parameter values are those assumed above):

$$
\lim _{g \rightarrow 0^{+}} l^{\prime}(g)=+\infty \quad \lim _{g \rightarrow+\infty} l^{\prime}(g)=0 \quad l^{\prime}(g)>0 \quad \forall g>0
$$


while the derivative of the right hand side is:

$$
r^{\prime}(g)=1
$$

As a consequence, the functions $l(g)$ and $r(g)$ coincide for $g=0$ (that is a solution, even if not interesting, of the equation considered), then the function $l(g)$ (that is strictly increasing for $g>0$ ) initially is steeper than the function $r(g)$ (which has a constant slope) and then it becomes flatter, and therefore the two functions coincide again for a (unique) value of $g>0$. This means that the solution (for $g>0$ ) of equation (64) exists and is unique.

The last aspect that can be considered is represented by the numerical simulation of the model when this specification for the productivity of schooling is assumed. Considering for instance the values $a=1.037$ and $b=0.007$ for the new parameters $a$ and $b$ that appear in this specification, the equation (64) is satisfied for $g=1.01105$ (other values of $a$ and $b$ can be considered, but the results of the simulations do not change in a substantial way). At this point it is possible to perform the simulations of the model for these parameter values (together with the values considered in Section 4 for the other parameters), and the results can be used in particular to derive the relationships between the growth factor of output $g_{Y}$ and the ratios $\frac{K}{H}, \frac{K}{R D}$ and $\frac{H}{R D}$. Considering first of all the relationship between the growth factor of output and the ratio between physical and human capital in this case we get:

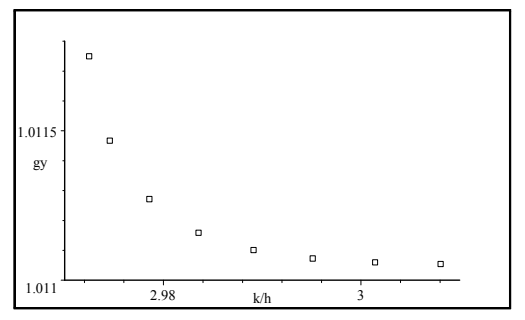

while considering the relationship between the growth factor of output and the ratio between physical capital and $\mathrm{R} \& \mathrm{D}$ expenditure we have:

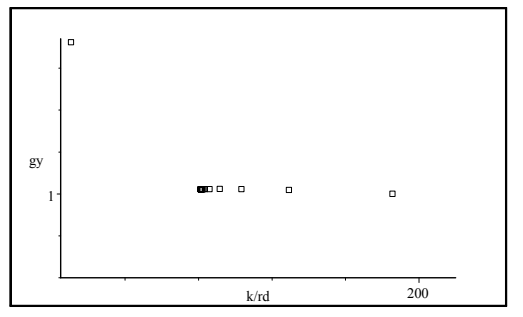


and similarly considering the relationship between the growth factor of output and the ratio between human capital and $R \& D$ expenditure we obtain:

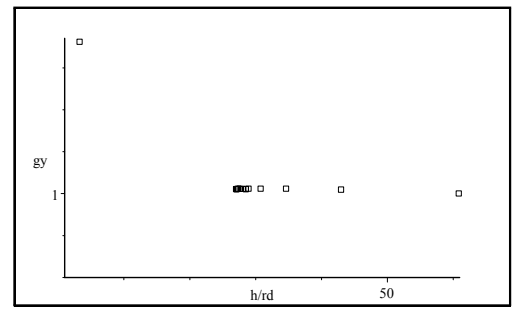

From these graphics it emerges that the imbalance effects continue to be present also in this version of the model (especially with reference to the behavior of the growth factor of output in relation to the ratio between physical and human capital, where the imbalance is particularly evident), and they are asymmetric, since the growth rate of output is higher when the ratio considered is below its steady state value, while it declines when this ratio is above its steady state value.

\section{Conclusions}

This paper has proposed a multi-sectoral endogenous growth model that reproduces an ICT-based economy considering R\&D activity with horizontal differentiation and human capital accumulation, in order to take into account the crucial role of the latter when new technologies are present. In this context, several results can be obtained, both analytically and through simulations. In particular, first of all the model doen't display any scale effect (since the long run growth rate of output doesn't depend neither on the absolute dimension of the economy nor on the population growth). A second result is represented by the fact that the productivity of schooling affects the long run growth of the economy, while the productivities of the other sectors don't have these effects, hence in this case human capital accumulation is the true engine of growth. A third result is that, in the model considered, there is a positive relationship beween market power and growth, contrary to other models of endogenous growth with horizontal differentiation but without human capital accumulation. Finally, imbalance effects with reference to some variables arise (in particular concerning the relationship between the growth rate of output on the one hand and the ratio physical capital/human 
capital, or physical capital/R\&D expenditure, or human capital/R\&D expenditure on the other hand), and this has important implications for the growth process. In the last part some extensions have been considered, in particular through the introduction of the notion of broad output, of different types of subsidies and of a link between human capital accumulation and technological progress. The results obtained show that imbalance effects arise also when the more general notion of broad output is considered or when a link between human capital and technological progress is assumed, and that the presence of subsidies on human capital accumulation has, in the short run, positive effects on the growth of the economy.

\section{References}

[1] Barro, R. and X. Sala-i-Martin (1995), "Economic Growth", McGrawHill.

[2] Boucekkine, R. and D. de la Croix (2003), "Information Technologies, Embodiment and Growth", Journal of Economic Dynamics and Control 27, 2007-2034.

[3] Gordon, R. (1999), "Has the New Economy Rendered the Productivity Slowdown Obsolete?", Mimeo, Northwestern University.

[4] Gordon, R. (2000), "Does the New Economy Measure up to the Great Inventions of the Past?", Journal of Economic Perspectives 14, 49-74.

[5] Greenwood, J., Z. Hercowitz and P. Krusell (1997), "Long-Run Implications of Investment-Specific Technological Change", American Economic Review 87, 342-362.

[6] Greenwood, J. and B. Jovanovic (1998), "Accounting for Growth", NBER Working Paper 6647.

[7] Greenwood, J. and B. Jovanovic (1999), "The IT Revolution and the Stock Market", American Economic Review Papers and Proceedings 89, 116-122.

[8] Greenwood, J. and M. Yorukoglu (1997), "1974", Carnegie-Rochester Conference Series on Public Policy 46, 49-95. 
[9] Hobijn, B. and B. Jovanovic (1999), "The Information Technology Revolution and the Stock Market: Preliminary Evidence", Mimeo, New York University.

[10] Jorgenson, D. and K. Stiroh (2000), "Raising the Speed Limit: US Economic Growth in the Information Age", Brookings Papers on Economic Activity 1, 125-212.

[11] Jovanovic, B. and P. Rousseau (2000), "Accounting for Stock Market Growth: 1885-1998", Mimeo, New York University.

[12] Juillard, M. (1996), "DYNARE, a Program for the Resolution of Non-Linear Models with Forward-Looking Variables. Release 2.1", CEPREMAP.

[13] Krusell, P. (1998), "Investment-Specific R\&D and the Decline in the Relative Price of Capital", Journal of Economic Growth 3, 131-141.

[14] Lucas, R. (1988), "On the Mechanics of Economic Development", Journal of Monetary Economics 22, 3-42.

[15] Mattalia, C. (2002), "Information Technologies, Economic Growth and Productivity Shocks", IRES Discussion Paper 2002-26, Université catholique de Louvain.

[16] Oliner, S. and D. Sichel (2000), "The Resurgence of Growth in the Late 1990's: Is Information Technology the Story?", Journal of Economic Perspectives 14, 3-22.

[17] Rivera-Batiz, L. and P. Romer (1991), "Economic Integration and Endogenous Growth", Quarterly Journal of Economics 106, 531-555.

[18] Romer, P. (1990), "Endogenous Technological Change", Journal of Political Economy 98, 71-102.

[19] Solow, R. (1960), "Investment and Technological Progress" in K.J. Arrow, S. Karlin and P. Suppes, Eds., Mathematical Methods in the Social Sciences 1959, Stanford University Press, 89-104.

[20] Whelan, K. (2000), "Computers, Obsolescence and Productivity", Mimeo, Federal Reserve Board, Division of Research and Statistics. 
Département des Sciences Économiques de l'Université catholique de Louvain

Institut de Recherches Économiques et Sociales

Place Montesquieu, 3

1348 Louvain-la-Neuve, Belgique 